\title{
Produção de snacks funcionais à base de farinha de soja e polvilho azedo
}

\author{
Production of functional snacks from soybean flour and sour cassava starch
}

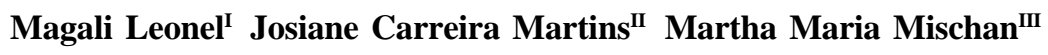

\section{RESUMO}

Este trabalho teve por objetivo avaliar o efeito da temperatura de extrusão e da porcentagem de farinha de soja sobre a expansão (IE), o índice de absorção de água (IAA), o índice de solubilidade em água (ISA), a cor ( $\left.L^{*}, a^{*} e b^{*}\right)$ e a dureza de biscoitos extrusados. O processo de extrusão seguiu o delineamento central composto rotacional para dois fatores, totalizando 11 tratamentos. Os resultados obtidos mostraram que a temperatura e a porcentagem de farinha de soja tiveram efeitos significativos sobre as propriedades físicas dos produtos. Nas condições intermediárias de temperatura e baixa porcentagem de farinha de soja (10\%), é possível obter biscoitos funcionais de mandioca com elevada expansão, índices intermediários de absorção e solubilidade em água, cor clara e baixa dureza.

Palavras-chave: amido, proteína, extrusão.

\section{ABSTRACT}

This study aimed to evaluate the effect of the extrusion temperature and the percentage of soybean flour in mixes on the expansion index (EI), water absorption index (WAI), water solubility index(WSI); color $\left(L^{*}, a^{*}, b^{*}\right)$ and hardness of extruded sour cassava snacks. The extrusion process followed the rotational central composite design for two factors, totalizing 11 treatments. The results showed that the extrusion temperature and percentage of soybean flour in mixes had significant effects on the physical properties of products. Under the conditions of intermediate temperature and low percentage of soybean meal is possible to obtain function cassava snacks with high expansion, intermediate index of absorption and solubility in water, light color and low hardness.

Key words: starch, protein, extrusion.

\section{INTRODUÇÃO}

O processo de extrusão é utilizado na produção de uma grande variedade de produtos, como cereais matinais, snacks, amidos modificados, produtos de confeitaria, proteínas vegetais texturizadas, produtos cárneos e rações animais. O processo de extrusão é definido como um processo contínuo, no qual ocorrem simultaneamente diversas operações, como a mistura, o cisalhamento, o cozimento e o modelamento (FELLOWS, 2002).

Nos últimos anos, a procura por alimentos derivados de soja tem aumentado em razão da divulgação dos benefícios à saúde atribuídos ao consumo dessa leguminosa. A soja é um alimento rico em proteínas, fibras, óleo, importante fonte de minerais (sódio, potássio, fósforo, ferro, magnésio, zinco e cálcio) e vitaminas, como tiamina $\left(\mathrm{B}_{1}\right)$, riboflavina $\left(\mathrm{B}_{2}\right)$, niacina $\left(\mathrm{B}_{3}\right)$, ácido nicotínico e ácido ascórbico. Além disso, produtos da soja desempenham função importante para a saúde, além de serem utilizados por pessoas alérgicas ao leite e também por suas boas características tecnológicas (BAZINET et al., 1997).

Na agroindustrialização da mandioca, um produto de importância e tradição é o polvilho azedo, amido de mandioca submetido à fermentação e secagem ao sol, que tem a propriedade de expansão como principal característica. É encontrado praticamente em todos os países da América do Sul, com exceção do Equador (PEREIRA et al., 1999).

ICentro de Raízes e Amidos Tropicais, Universidade Estadual Paulista “Júlio de Mesquita Filho” (UNESP), 18610-307, Botucatu, SP, Brasil. E-mail: mleonel@fca.unesp.br. Autor para correspondência.

${ }^{\mathrm{II} C u r s o ~ d e ~ N u t r i c ̧ a ̃ o, ~ I n s t i t u t o ~ d e ~ B i o c i e ̂ n c i a s, ~ U N E S P, ~ B o t u c a t u, ~ S P, ~ B r a s i l . ~}$

IIIDepartamento de Bioestatística, Instituto de Biociências, UNESP, Botucatu, SP, Brasil 
O polvilho azedo apresenta viscosidade e temperatura inicial de pasta menores que as do polvilho doce e sem tendência a geleificação durante o resfriamento. GUYOT \& MORLON-GUYOT (2001) afirmam que essas modificações produzidas no polvilho azedo, durante o processo fermentativo, são as que conferem ao produto assado aparência inchada, estrutura típica alveolar do miolo e da crosta crocante.

Tendo em vista a expressão da cultura da mandioca na economia brasileira, o forte apelo social, bem como a grande aceitação do biscoito de polvilho azedo, este trabalho avaliou o efeito da temperatura de extrusão e da porcentagem de farinha de soja na produção de biscoitos funcionais de polvilho azedo.

\section{MATERIAL E MÉTODOS}

Foram preparadas misturas de $700 \mathrm{~g}$ de polvilho azedo e farinha de soja em batedeira planetária, nas porcentagens de farinha de soja do delineamento experimental (Tabela 1). Após a mistura das matériasprimas, foi realizado o processo de extrusão em linha completa de extrusão IMBRA RX da Inbramaq S/A, com capacidade de produção de $45 \mathrm{~kg} \mathrm{~h}^{-1}$.

O processo seguiu o delineamento "central composto rotacional" para dois fatores, segundo BARROS NETO et al. (2007), com um total de 11 tratamentos (Tabela 1). Os parâmetros fixos do processo foram: umidade das misturas (12\%), taxa de compressão da rosca (3:1), abertura da matriz (3mm), taxa de alimentação $\left(180 \mathrm{~g} \mathrm{~min}^{-1}\right)$, rotação da rosca (272rpm) e temperatura na $1^{\underline{a}}$ zona $\left(25^{\circ} \mathrm{C}\right)$ e na $2^{\underline{a}}$ zona $\left(40^{\circ} \mathrm{C}\right)$ do canhão de extrusão.

O índice de expansão dos extrusados foi calculado pela relação entre o diâmetro da amostra e o diâmetro da matriz. O valor considerado foi obtido pela média aritmética das medidas de 15 diferentes produtos expandidos dentro de cada tratamento.

A determinação do índice de absorção de água antes e após os tratamentos de extrusão seguiu a

Tabela 1 - Parâmetros variáveis do processo de extrusão.

\begin{tabular}{cccc}
\hline & & \multicolumn{2}{c}{$\begin{array}{c}\text { Fatores ou variáveis } \\
\text { independentes }\end{array}$} \\
\hline Axiais & Codificados & $\mathrm{T}$ & $\mathrm{FS}$ \\
$-\alpha$ & $-1,41$ & 61,4 & 5,7 \\
& -1 & 70 & 10 \\
& 0 & 90 & 20 \\
$+\alpha$ & +1 & 110 & 30 \\
& $+1,41$ & 118,6 & 34,3 \\
\hline
\end{tabular}

T: Temperatura de extrusão $\left({ }^{\circ} \mathrm{C}\right)$; FS: Farinha de soja (\%). metodologia de ANDERSON et al. (1969). O IAA foi calculado conforme a seguinte relação: IAA=PRCx100/ PA-PRE, em que: $P R C=$ peso de resíduo de centrifugação (g); PA = peso da amostra (base seca); e $\mathrm{PRE}=$ peso de resíduo se evaporação $(\mathrm{g})$

O ISA foi calculado pela relação entre o peso do resíduo de evaporação e o peso da amostra (ANDERSON et al.,1969).

A cor das misturas de polvilho azedo e farinha de soja e dos produtos extrusados foi avaliada em colorímetro Minolta CR-400. Os resultados foram expressos em valores de $L^{*}, a^{*}$ e b*.

A determinação da dureza (parâmetro de textura) foi realizada em analisador de textura LFRABrookfield de carga máxima de $25 \mathrm{~kg}$, um dispositivo de teste cilíndrico com extremidade plana. Os parâmetros utilizados para a avaliação das amostras foram: velocidade pré-teste de $2,5 \mathrm{~mm} \mathrm{~s}^{-1}$, velocidade teste de $1,0 \mathrm{~mm} \mathrm{~s}^{-1}$ e pós-teste de $4,0 \mathrm{~mm} \mathrm{~s}^{-1}$.

Para a análise estatística dos resultados experimentais, foi utilizado o modelo:

$\mathrm{y}_{\mathrm{k}}=\beta_{0}+\beta_{1} \mathrm{x}_{1}+\beta_{2} \mathrm{x}_{2}+\beta_{12} \mathrm{x}_{1} \mathrm{x}_{2}+\varepsilon$

em que

$\mathrm{y}_{\mathrm{k}}=$ valor observado da variável dependente no nível

$K, \mathrm{~K}=1, \ldots, 11$;

$\beta_{0}=$ valor populacional da média de todas as respostas do planejamento;

$\beta_{1}, \beta_{2}, \beta_{12}=$ valores populacionais dos dois efeitos principais e do efeito da interação, por unidade de $\mathrm{x}_{1} \mathrm{e}$ $\mathrm{x}_{2}$; $\mathrm{e}$

$\varepsilon=$ erro aleatório associado à resposta y $\left(\mathrm{x}_{1}, \mathrm{x}_{2}\right)$.

$\mathrm{O}$ processamento dos dados e a análise estatística foram realizados com o auxílio do sistema SAS. O ajuste do modelo foi feito pela opção stepwise, e o modelo ajustado obtido foi validado pelo teste F, sendo utilizado como denominador o quadrado médio do erro puro (BARROS NETO et al., 2007).A significância do modelo foi testada pela análise de variância (ANOVA), sendo adotado o nível de significância alfa de 5\%. Na construção das superfícies de resposta, duas variáveis independentes variaram dentro das regiões estudadas.

\section{RESULTADOS E DISCUSSÃO}

Por meio dos resultados experimentais obtidos na caracterização dos produtos extrusados, foi possível determinar os coeficientes de regressão para as variáveis dependentes, sendo apresentados na tabela 2 os modelos codificados.

A formação de produtos expandidos, com forma e textura desejáveis, depende de inúmeros fatores. Os mais importantes são a gelatinização 
Tabela 2 - Coeficientes de regressão (modelo $y_{k}=\beta_{0}+\beta_{1} x_{1}+\beta_{2} x_{2}+\beta_{12} x_{1} x_{2}$ ).

\begin{tabular}{|c|c|c|c|c|c|c|c|}
\hline \multirow{2}{*}{ Parâmetros } & \multicolumn{7}{|l|}{ 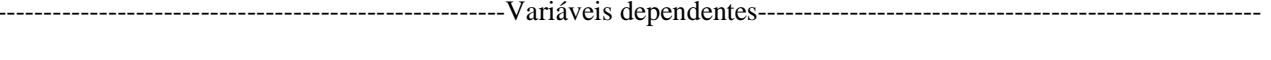 } \\
\hline & IE & IAA & ISA & $\mathrm{L}^{*}$ & $a^{*}$ & $b^{*}$ & DU \\
\hline$\beta_{0}$ & 3,35 & 7,83 & 62,45 & 74,36 & 4,69 & 22,12 & 3,7828 \\
\hline$\beta_{1}$ & $-0,22 * *$ & $-0,29 *$ & $2,58^{*}$ & $-1,09$ & $0,43^{*}$ & 0,32 & $-0,2257$ \\
\hline$\beta_{2}$ & $-0,26 * * *$ & $-1,20 * * *$ & $5,81 * *$ & $-1,34$ & 0,11 & $2,07 * *$ & $0,5415^{* *}$ \\
\hline$\beta_{11}$ & $0,22 * *$ & $-0,22$ & 2,52 & $-0,23$ & $-0,16$ & $-0,17$ & $0,5412 *$ \\
\hline$\beta_{22}$ & 0,09 & $0,36^{*}$ & $-1,82$ & 0,66 & $-0,35$ & 0,73 & 0,3601 \\
\hline$\beta_{12}$ & 0,03 & $0,46^{*}$ & $-1,11$ & $-0,95$ & 0,45 & 0,14 & 0,5125* \\
\hline $\mathrm{R}^{2}$ & 0,9641 & 0,9718 & 0,9101 & 0,6710 & 0,7561 & 0,8206 & 0,8796 \\
\hline $\operatorname{Pr}>F$ & 0,0013 & 0,0007 & 0,0119 & 0,2264 & 0,1200 & 0,0604 & 0,0239 \\
\hline
\end{tabular}

$\beta_{1}=$ temperatura (linear); $\beta_{2}=$ farinha de soja (linear); $\beta_{11}=$ temperatura (quadrático); $\beta_{22}=$ farinha de soja (quadrático); $\mathrm{R}^{2}=$ coeficiente de determinação; $*=\mathrm{P}<0,05, * *=\mathrm{P}<0,01, * * *=\mathrm{P}<0,001$; IE=índice de expansão; IAA=índice de absorção de água g gel $\mathrm{g}^{-1}$; ISA=índice de solubilidade em água (\%); L*=luminosidade; $\mathrm{a}^{*}=$ croma $\mathrm{a}^{*}$; b*=croma b*; DU=dureza (kgf).

adequada do amido e o desenvolvimento de zonas de alta pressão próximas à matriz, para causar rápida evaporação da água e formação de uma estrutura celular forte (CAMIRE et al., 1990).

O índice de expansão dos produtos extrusados variou de 3,18 a 4,17. O tratamento com menor IE foi o que apresentava maior porcentagem de soja $(34,3 \%)$ na mistura com polvilho azedo. Esse resultado já era esperado em razão do efeito das proteínas, dos lipídeos e das fibras, presentes em quantidades significativas na farinha de soja, sobre a expansão de produtos extrusados.

A análise dos coeficientes de regressão mostrou efeito linear e quadrático da temperatura de extrusão, bem como efeito linear da porcentagem de farinha de soja na mistura sobre o índice de expansão.

A superfície de resposta demonstra que valores elevados de temperatura de extrusão e porcentagem de farinha de soja levam à obtenção de produtos extrusados de baixa expansão. Na condição de menor teor de farinha de soja e baixa temperatura, a expansão é elevada, já na condição de elevada temperatura e alta porcentagem de farinha de soja a expansão é menor (Figura 1).

O grau de expansão radial é proporcional à temperatura até certo valor para depois decrescer em temperaturas elevadas. A diminuição da expansão em temperaturas elevadas é atribuída ao aumento da dextrinização e enfraquecimento da estrutura. Já quanto ao efeito da farinha de soja, de acordo com MERCIER et al. (1998), o grau de expansão máxima está relacionado ao conteúdo de amido, sendo relatado um aumento de 500\% no diâmetro do produto obtido de amidos puros.

CAMARGO et al. (2008), estudando o efeito da umidade, temperatura e porcentagem de fibra no desenvolvimento de biscoitos de polvilho azedo extrusados, verificaram uma variação do índice de expansão de 3,5 a 5,7, e os maiores valores de volume específico (variação de 1,5 a 5,6mL g-1 ) ocorreram nas condições em que não foi adicionado farelo e o teor de umidade inicial do material era baixo.

CHANG et al. (2001) estudaram os efeitos da adição de isolado proteico de soja (0-50\%), umidade inicial (17,96-28,04\%) e temperatura de processo (106,4$\left.173,6^{\circ} \mathrm{C}\right)$ no desenvolvimento de snack expandido à base de amido de mandioca e concluíram que a expansão aumentou com temperaturas maiores de processo e alto conteúdo de isolado protéico. Esses resultados diferem dos obtidos neste experimento com polvilho azedo, contudo é importante ressaltar que a umidade da mistura foi baixa (12\%), o que promove maior atrito e, consequentemente, o efeito da temperatura de extrusão sobre os componentes da matéria-prima é mais acentuado.

O índice de absorção de água (IAA) está relacionado com a disponibilidade de grupos hidrofílicos $(-\mathrm{OH})$ em se ligar às moléculas de água e capacidade de formação de gel das moléculas de amido. Os índices de absorção de água das misturas de polvilho azedo e farinha de soja antes do processo de extrusão variaram de 1,95 a 2,18g gel g-1 , da menor para a maior porcentagem de farinha de soja, respectivamente. Segundo GARCIA et al. (1997), as principais propriedades funcionais da proteína de soja são: capacidade de retenção de água, emulsificação, gelatinização e formação de espuma, o que pode explicar o aumento do IAA das misturas com o aumento da porcentagem de farinha de soja. Após o processo de extrusão, os resultados obtidos nos diferentes tratamentos mostram uma variação de 6,33 a 10,31g gel 


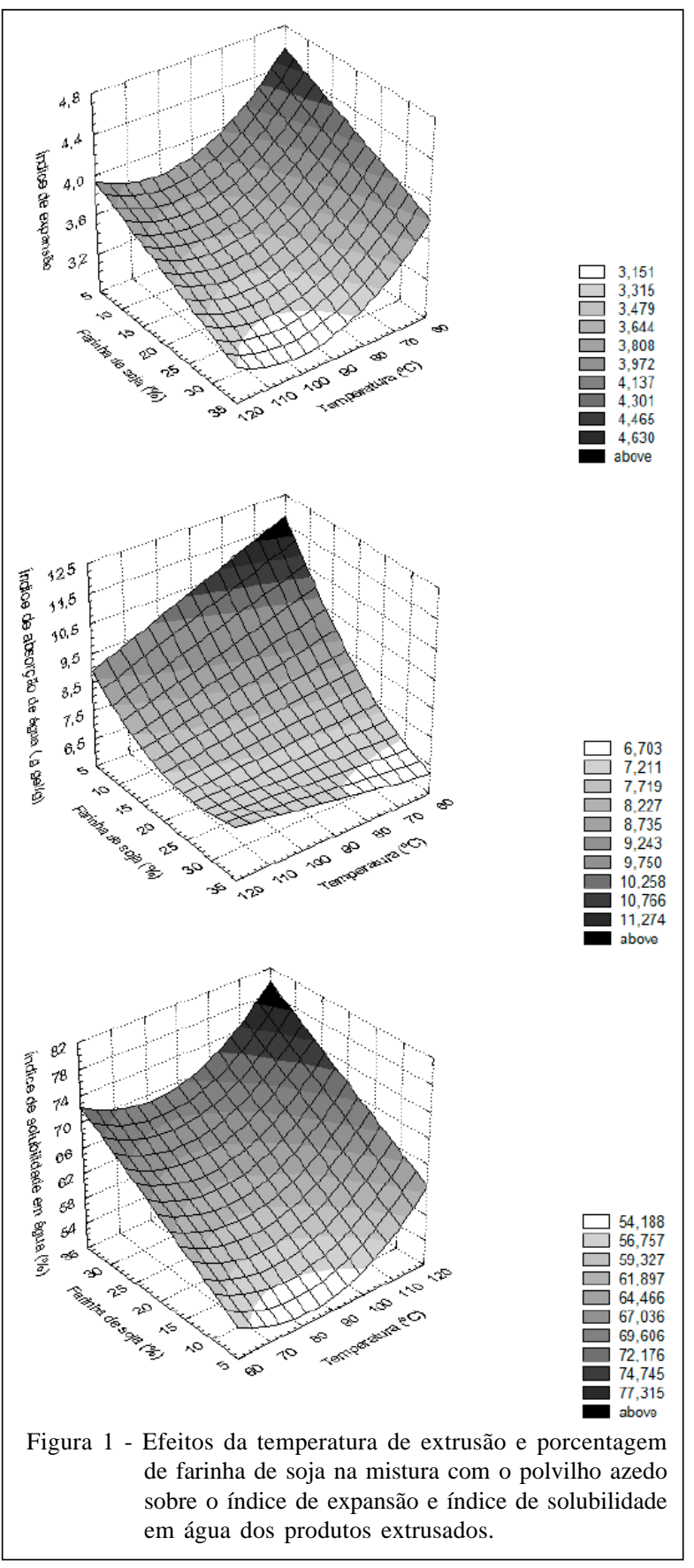

$\mathrm{g}^{-1}$, ou seja, o processo de extrusão aumentou a absorção de água independente das condições experimentais.

A análise dos coeficientes de regressão mostrou que ocorreram efeito linear da temperatura de extrusão, linear e quadrático da porcentagem de farinha de soja e interação dos dois fatores sobre o IAA dos produtos extrusados. Os maiores valores de IAA são obtidos nas condições de menor temperatura de extrusão e menor porcentagem de farinha de soja. Já o aumento do teor de proteína unido à baixa temperatura leva a menores IAA (Figura 1).

Baixas temperaturas permitem maiores cadeias de polímeros não danificados e uma grande disponibilidade de grupos hidrolíticos, aos quais se ligam mais moléculas de água, resultando em altos valores de IAA (SOUZA et al., 2007).

Durante a extrusão, os grânulos de amido sofrem gelatinização, e as proteínas são desnaturadas. $\mathrm{O}$ amido gelatinizado absorve mais água do que em seu estado natural e as proteínas, devido a mudanças conformacionais e estruturais, têm o seu balanço hidrofílico/hidrofóbico alterado, podendo contribuir para o aumento ou diminuição do IAA (ALVIM et al., 2002).

O índice de solubilidade em água normalmente é utilizado em produtos extrusados como indicador da degradação dos componentes moleculares. O ISA das misturas de polvilho azedo e farinha de soja, nas diferentes porcentagens desta, variaram de 2,01 a 7,34\% antes do processo de extrusão, sendo o maior valor obtido na mistura com maior porcentagem de farinha de soja (34,3\%). Após o processo de extrusão, verificou-se uma variação de 51,39 a 73,74\%, valores bastante elevados quando comparados aos obtidos antes da extrusão.

A análise dos dados mostrou terem ocorrido efeito linear e efeito quadrático da temperatura de extrusão, bem como efeito linear da porcentagem de farinha de soja sobre a solubilidade em água dos produtos extrusados. Os menores valores de solubilidade em água, característica desejável para produtos expandidos, são obtidos nas condições de temperatura baixa à intermediária e baixo teor de farinha de soja. Nas condições de elevada porcentagem de farinha de soja e alta temperatura de extrusão, o ISA é maior (Figura 1).

O ISA depende da intensidade e do tipo de reações que ocorrem durante a extrusão, influenciando a quantidade de moléculas solúveis. Além da gelatinização do amido, que resulta na liberação de amilose e amilopectina, pode ocorrer também a dextrinização dos componentes do amido e outras reações que conduzem à formação de compostos de baixo peso molecular (RIAZ, 2000).

Durante a extrusão, as proteínas nativas passam por uma total desagregação, com a fusão em 
uma suspensão homogênea que propicia a desnaturação, dissociação e formação de filamentos que se alinham no sentido do fluxo até a expulsão da máquina. Nas transformações químicas da desnaturação, as forças de estabilização das estruturas terciárias e quaternárias da proteína são enfraquecidas pela combinação da temperatura alta e da compressão dentro do extrusor. São rompidas ligações iônicas, dissulfeto, pontes de hidrogênio e interações fracas, do tipo Van der Waals. Com essa desnaturação, surgem proteínas com diferentes pesos moleculares, bem como aminoácidos até então indisponíveis, o que interfere no ISA.

A cor é uma qualidade visual (atributo) importante dos produtos alimentícios. Existem muitas reações que ocorrem durante a extrusão que afetam a cor. Entre elas, as mais comuns são as reações de escurecimento não enzimático (reação de Maillard e caramelização) e a degradação de pigmentos (ILO \& BERGHOFER, 1999).

A luminosidade dos produtos extrusados variou de 70,35 a 77,96, valores inferiores aos obtidos na análise das misturas de polvilho azedo e farinha de soja antes do processo de extrusão, os quais variaram de 89,71 a 93,85, da maior para a menor porcentagem de farinha de soja na mistura.

A análise estatística dos dados mostrou não terem ocorrido efeitos dos fatores temperatura de extrusão e porcentagem de farinha de soja para esse parâmetro de cor; contudo, a menor luminosidade foi observada na condição de maior temperatura de extrusão testada.

Para o croma a*, observou-se uma variação de 0,31 a 0,70 nas misturas antes do processo de extrusão, com o maior valor obtido na mistura com maior porcentagem de farinha de soja. Nos produtos extrusados, ocorreu uma variação de 3,7 a 5,31 para esse componente de cromaticidade. A análise dos coeficientes de regressão evidenciou efeito linear da temperatura de extrusão sobre o croma $\mathrm{a}^{*}$. O aumento da temperatura acarretou aumento do croma $\mathrm{a}^{*}$.

A análise do componente de cromaticidade b* nas misturas antes do processo de extrusão mostrou uma variação de 9,23 a 15,61, com maior valor na mistura com 34,3\% de farinha de soja. Após a extrusão, nas diferentes condições experimentais, os produtos extrusados apresentaram valores de croma $b^{*}$ variando de 19,23 a 27,94, evidenciando a ocorrência de reações de escurecimento.
Com relação ao efeito dos fatores do processo sobre esse parâmetro de cor, a análise dos coeficientes de regressão mostrou ter ocorrido efeito linear da porcentagem de farinha de soja sobre esse parâmetro. O aumento do croma b* é diretamente proporcional ao aumento da porcentagem de farinha de soja na mistura com o polvilho azedo, o que já havia sido observado nas misturas antes do processo de extrusão.

Um parâmetro de qualidade de grande importância na aceitação de produtos extrusados é a dureza, parâmetro de textura que fisicamente representa a força necessária para produzir certa deformação; enquanto que, sensorialmente, representa a força necessária para a compressão de uma substância entre os dentes (MEILGAARD et al., 1999). A dureza dos biscoitos de polvilho e soja extrusados variou de 3,58 a 5,9kgf.

A análise dos coeficientes de regressão mostrou ter ocorrido efeito linear da porcentagem de farinha de soja na mistura, efeito quadrático da temperatura de extrusão e efeito da interação dos fatores sobre a textura dos produtos extrusados.

$\mathrm{O}$ que se deseja em snacks com boas características de textura é que os valores de dureza sejam baixos. De acordo com a superfície de resposta traçada a partir do modelo ajustado, biscoitos com menor dureza são obtidos nas condições de elevada temperatura e baixas porcentagens de farinha de soja na mistura com o polvilho azedo (Figura 2).

\section{CONCLUSÃO}

A partir dos resultados obtidos, foi possível concluir que a mistura do polvilho azedo com a farinha de soja pode ser utilizada na produção de biscoito funcional de polvilho azedo, sendo possível a obtenção das características desejadas para esse tipo de produto, como elevada expansão, índices intermediários de absorção e solubilidade em água, cor clara e menor dureza, quando o processo ocorre em condições intermediárias de temperatura na zona de extrusão e menor porcentagem de farinha de soja.

\section{AGRADECIMENTO}

Os autores agradecem o auxílio financeiro da Fundação de Apoio à Pesquisa do Estado de São Paulo (FAPESP).

Ciência Rural, v.40, n.6, jun, 2010. 


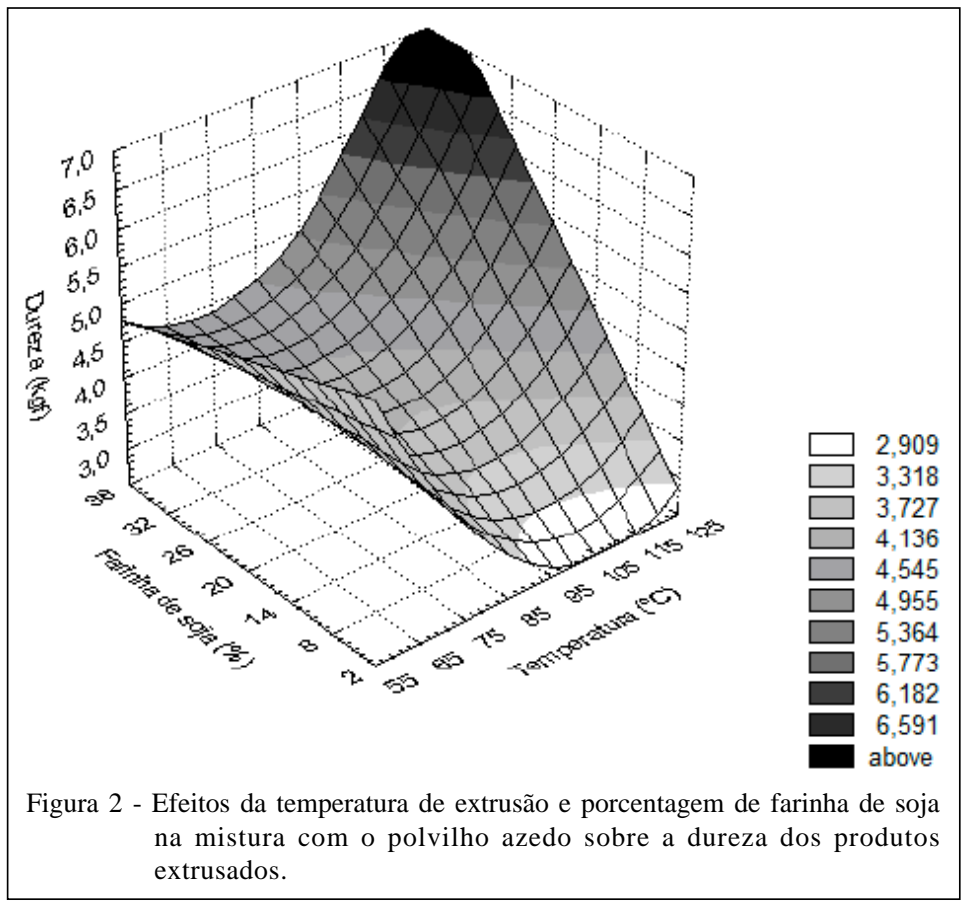

\section{REFERÊNCIAS}

ALVIM, I.D. et al. Desenvolvimento de farinhas mistas extrusadas à base de farinha de milho, derivados de levedura e caseína. Ciência e Tecnologia de Alimentos, Campinas, v.22 p.170-176, 2002. Disponível em: <http://www.scielo.br/ scielo.ph p ? s c ript = sci_art text \& pid = S 0101 20612002000200012\&lng=pt\&nrm=iso>. Acesso em: 22 jan. 2009. doi: 10.1590/S0101-20612002000200012.

ANDERSON, R.A. et al. Gelatinization of corn grits by rolland extrusion-cooking. Cereal Science Today, St Paul, v.14, n.1, p.4-7, 1969.

BARROS NETO, B. et al. Como fazer experimentos Pesquisa e desenvolvimento na ciência e na indústria. 3.ed. Campinas: Unicamp, 2007. 480p.

BAZINET, L. et al. Electroacidification of soybean for production of isolate. Food Technology, Chicago, v.51, n.9, p.52-60, 1997.

CAMARGO, K.F. et al. Produção de biscoitos extrusados de polvilho azedo com fibras: efeito de parâmetros operacionais sobre as propriedades físicas. Ciência e Tecnologia de Alimentos, Campinas, v.28, n.3, p.586-591, 2008. Disponível em: <http:// www.scielo.br/scielo.php?script=sci_arttext \&pid=S010120612008000300013\&lng=pt\&nrm=iso $>$. Acesso em: 16 out. 2009. doi: 10.1590/S0101-20612008000300013.

CAMIRE, M.E. et al. Chemical and nutritional changes in foods during extrusion. Critical Reviews in Food Science and Nutrition, Philadelphia, v.19, p.35-57, 1990.

CHANG, Y.K. et al . Influence of extrusion condition on cassava starch and soybean protein concentrate blends. Acta Alimentaria, Budapest, v.30, n.2, p.189-203, 2001.
FELLOWS, P. Extrusion. In: FELLOWS, P. Food processing technology: principles and practive. Cambridge: Woodhead Publishing, 2002. Cap.14, p.294308 .

GARCIA, M.C. et al. Composition and characterization of soybean and related products. CRC Critical Reviews in Food Science and Nutrition, Boca Raton, v.37, n.4, p.361-391, 1997. Disponível em: <http:/ / w w w. in form a w or ld.com/ s m p p / ftinterface content=a915431194 fulltext=713240930>. Acesso em: 19 jan. 2010.

GUYOT, J.P.; MORLON-GUYOT, J. Effect of different cultivation conditions on Lactobacillus manihotivorans OND $32^{\mathrm{T}}$, an amylolitic lactobacillus isolated from sour cassava fermentation. International Journal of Food Microbiology, Oxford, v.67, p.217-225, 2001. Disponível em: <http:// w w w. s c i e n c e d i r e c t . c o m/ science?_ob=MImg\&_imagekey=B6T7K$\begin{array}{lllllllll}4 & 3 & \mathrm{~J} & 7 & \mathrm{X} & \mathrm{W} & \mathrm{G} & - & 5\end{array}$ C\&_cdi $=5061 \&$ \&_user $=972052 \&$ \& ii $=$ S0 16 $8160501004445 \&$ \& orig = search \&_co ver Date $=08 \% 2$ F $05 \% 2$ F $2001 \&$ \& s $\mathrm{k}=999329996$ \& vi e w $=\mathrm{c} \& \mathrm{w}$ c h p $=$ d G L z V t z zSkzk\&md5=d0ccac97ecf597092755d6308af19c9f\&ie=/ sdarticle.pdf $>$. Acesso em: 19 jan. 2010.

ILO, S.; BERGHOFER, E. Kinetics of colour changes during extrusion cooking of maize grits. Journal of Food Engineering, Oxford, v.39, n.1, p.73-80, 1999. Disponível em: <http:// www.sciencedirect.com/science?_ob=MImg\&_imagekey=B6T8J40 M52Y F-B-H\&_cdi $=5088 \&$ \&_user $=972052 \&$ \& ii $=$ S 0 260877498001484 \&_orig = s e a r c h \&_c o verDate=01\%2F31\%2F1999\&_sk=999609998\&view=c\&wchp=dGLbVtzzSkzk\&md5=cb88142c60aea798833c9641de137068\&ie $=$ / sdarticle.pdf>. Acesso em: 16 out. 2009.

MEILGAARD, M. et al. Sensory evaluation techniques. 3.ed. Boca Raton: CRC, 1999. 387p.

MERCIER, C. et al. Extrusion cooking. 2.ed. St. Paul: American Association of Cereal Chemists, 1998. 471p.

PEREIRA, J. et al. Féculas fermentadas na fabricação de biscoitos: estudo de fontes alternativas. Ciência e Tecnologia de Alimentos, Campinas, v.19, n.2, p.287-293, 1999. Disponível em: <http:// www.scielo.br/scielo.php?script=sci_arttext\&pid=S010120611999000200024\&lng=pt\&nrm=iso>. Acesso em: 09 nov. 2009. doi: 10.1590/S0101-20611999000200024.

RIAZ, M.N. Extruders in Food Applications. Boca Raton, FL: CRC, 2000. 225p.

SOUZA, L.B. et al. Efeitos dos parâmetros de extrusão nas propriedades físicas de extrusados de misturas de fécula de mandioca e polpa cítrica. Alimentos e Nutrição, Araraquara, v.18, p.83-91, 2007. Disponível em: http://servbib.fcfar.unesp.br/seer/index.php/alimentos/article/view/138/ 147. Acesso em: 21 out. 2009. 